\title{
Reactive oxygen species may influence on the crossroads of stemness, senescence, and carcinogenesis in a cell via the roles of APRO family proteins
}

\author{
Yuka Ikeda, Kurumi Taniguchi, Nozomi Nagase, Ai Tsuji, Yasuko Kitagishi, Satoru Matsuda* \\ Department of Food Science and Nutrition, Nara Women's University, Kita-Uoya Nishimachi, Nara 630-8506, Japan \\ *Correspondence: Satoru Matsuda, Department of Food Science and Nutrition, Nara Women's University, Kita-Uoya \\ Nishimachi, Nara 630-8506, Japan. smatsuda@cc.nara-wu.ac.jp \\ Academic Editor: Esma R. Isenovic, University of Belgrade, Serbia \\ Received: July 30, 2021 Accepted: August 30, 2021 Published: October 31, 2021
}

Cite this article: Ikeda Y, Taniguchi K, Nagase N, Tsuji A, Kitagishi Y, Matsuda S. Reactive oxygen species may influence on the crossroads of stemness, senescence, and carcinogenesis in a cell via the roles of APRO family proteins. Explor Med. 2021;2:443-54. https://doi.org/10.37349/emed.2021.00062

\begin{abstract}
Excessive reactive oxygen species (ROS) may cause oxidative stress which is involved in aging and in the pathogenesis of various human diseases. Whereas unregulated levels of the ROS may be harmful, regulated basal level of ROS is even necessary to support cellular functions as a second messenger for homeostasis under physiological conditions. Therefore, redox medicine could develop as a new therapeutic concept for human health-benefits. Here, we introduce the involvement of ROS on the crossroads of stemness, senescence, and carcinogenesis in a stem cell and cancer cell biology. Amazingly, the anti-proliferative (APRO) family anti-proliferative proteins characterized by immediate early growth responsive genes may also be involved in the crossroads machinery. The biological functions of APRO proteins (APROs) seem to be quite intricate, however, which might be a key modulator of microRNAs (miRNAs). Given the crucial roles of ROS and APROs for pathophysiological functions, upcoming novel therapeutics should include vigilant modulation of the redox state. Next generation of medicine including regenerative medicine and/or cancer therapy will likely comprise strategies for altering the redox environment with the APROs via the modulation of miRNAs as well as with the regulation of ROS of cells in a sustainable manner.
\end{abstract}

\section{Keywords}

APRO family, stem cell, cancer, stemness, senescence, carcinogenesis, reactive oxygen species

\section{Introduction}

Reactive oxygen species (ROS) include a set of highly reactive radicals such as superoxide anion and hydroxyl radical as well as non-radical species such as hydrogen peroxide, largely derived from oxidative metabolism in cellular physiological processes $[1,2]$. Main intracellular source of ROS is the mitochondrial electron transport chain during the ATP-synthesis in mammals [3]. Unnecessary ROS production may cause oxidative stress which is involved in aging and also in the pathogenesis of various human diseases including 
cancer [4]. The function of ROS as intracellular second messengers and as extracellular mediators is leading physiological signaling [5]. While ROS may also affect various pathophysiological conditions, they will be required as selective strategies for therapeutic intervention. Redox medicine is a new therapeutic concept targeting ROS for human health benefit. In particular, stem cells are responsible for regeneration supporting homeostasis of an organism through self-renewal, proliferation, and differentiation, whose function can be affected by ROS [6]. Initially, ROS were considered to act as molecules damaging to cellular components [7]. For example, high ROS levels are known to cause cellular DNA damages. However, many studies have accepted that ROS play a critical role in physiological processes in lower ROS levels [8]. In addition, different degrees of ROS would affect cell energetics and intracellular signaling pathways to regulate messenger RNA (mRNA) and protein expression, which determines cell fate modulating either cell survival or cell death [9]. Interestingly, ROS mediated significant responses including stemness of stem cells, cellular senescence, and carcinogenesis seem to be involved in the roles of anti-proliferative (APRO) family proteins [10]. In fact, expression of an APRO protein is frequently downregulated in many human cancers and in stem cells [11]. Furthermore, the APRO proteins have been implicated in a variety of cellular processes including cell division, DNA repair, transcriptional regulation and mRNA stability, which could regulate cell-cycle progression, apoptosis, and/or differentiation [12]. Therefore, APRO proteins might be a link between cellular senescence and carcinogenesis [10]. In this review, we would like to highlight the molecular mechanisms and biological consequences of APRO family proteins during stem cell-maintenance, senescence, and cancer progression with ROS motivations for the future superior medical application.

\section{ROS, stem cells and APRO family}

Stem cells are characterized by their ability to differentiate into several specific cells, which retain a high proliferative capability and plasticity [13]. In addition, stem cells are robust to collapse and protected against the responses to various stimulus [14]. However, ROS stimuli have been hypothesized to lead to the loss of the transplanted stem cells [15]. In general, ROS may inhibit the proliferation of stem cells and enhance the differentiation to the specific cells [16] (Figure 1). The impacts of ROS on stem cells proliferation and/or differentiation have attracted interests due to potential applications of stem cells for medical regenerative therapeutics. Antioxidant enzymes such as catalase and/or superoxide dismutase (SOD) have been shown upregulated upon the stem cells differentiation, which led to a dramatic decrease in the intracellular ROS level [17-19]. ROS metabolism might be definitely controlled by various antioxidant molecules involved in the redox machinery in cells. One mechanism by which ROS may exert their effects is via the regulation of target molecules in a phosphoinositide-3 kinase (PI3K)/AKT pathway [20]. Since stem cells are firmly regulated by oxidative stress, the control of ROS levels is important to maintain their self-renewal capability. In quiescent stem cells, very low levels of ROS may be indispensable for their stemness-maintenance; however, physiological ROS levels may encourage stem cells' proliferation and/or differentiation [21]. High levels of ROS are mostly involved in programmed cell death and/or apoptosis [22]. For example, radiation therapy kills cancer cells by generating high levels of ROS, which contributes to inducing severe DNA damages and cancer cells death. Remarkably, members of APRO family transducer of ErbB-2 (TOB)1 and TOB2 may inhibit the proliferation of mouse embryonic stem cells [23]. It has been shown that embryonic stem cells in single knockout $\mathrm{TOB} 1 \%$ or $\mathrm{TOB} 2 \%$, and double knockout $\mathrm{TOB} 1 \%$ and $\mathrm{TOB} 2 \%$ grow faster than wild type embryonic stem cells without losing pluripotency [23]. In addition, knockdown of TOB1 considerably increases the proliferative activity of mesenchymal rat stem cells in vitro [24]. B-cell translocation gene (BTG)1, the other member in APRO family, also seems to be necessary for maintaining stem cells' quiescence and self-renewal [25]. Downregulation of Nanog has resulted in reduction of pluripotency markers such as Krüppel-like factor 2 (Klf2) and octamer-binding transcription factor 4 (Oct-4) in P19 stem cells, whereas expression of TOB1 has been upregulated by the Nanog-silencing [26]. In addition, another APRO member tetradecanoyl phorbol acetate-inducible sequences (TIS)21/BTG2 plays a pivotal role in maintaining the hematopoietic stem cells' compartment and/or hematopoiesis [27]. Furthermore, physical exercise in BTG1-null mice may rescue the loss of proliferative capability arising in elder stem cells $[11,28]$. 


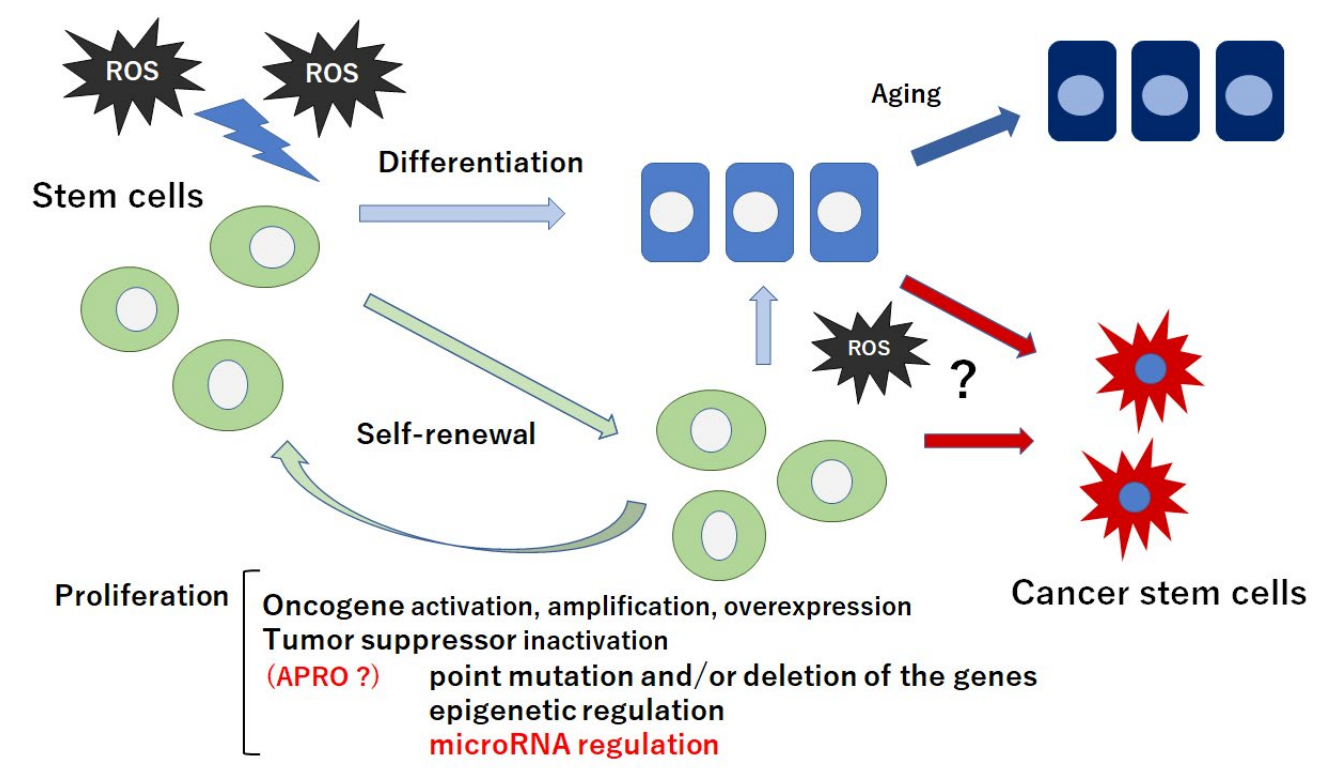

Figure 1. Schematic representation of stem cells self-renewal, proliferation, and differentiation. Stem cells and/or cancer stem cells are capable of maintaining the stem cells population through appropriate self-renewal and proliferation. Quiescent and/or self-renewing stem cells exhibit low ROS levels probably due to their robust antioxidant ability. Intermediate levels of ROS may prime stem cells for differentiation and/or cancer stem cell-formation. Tumor suppressor inactivation by microRNA-regulation could lead to the proliferation of stem cells and/or cancer stem cells. Note that some critical pathways have been omitted for clarity. ?: the phenomena have not yet been confirmed at present

\section{ROS, senescence and APRO family}

During cellular aging, boosted ROS generation and reduced antioxidants may result in redox imbalance triggering age-related diseases [29]. ROS could weaken cellular function via the modification of proteins and lipids in cells and/or tissues [30]. For example, ROS-induced lipid peroxidation occurs in physical dysfunction in elderly individuals [31]. Consequently, aging may be connected with the accumulation of oxidized molecules categorized by elevated carbonyl residues [32, 33]. Enhanced oxidative stress-mediated protein carbonyls may contribute to age-related diseases [34]. Being declined antioxidant defense by aging, fragility to remove oxidative-damaged molecules could probably accelerate the aging further. Also, long-term exposure to oxidative stress conditions is harmful. It has been shown that chronic oxidative modification of proteins leads to protein aggregation, diminished cellular function, and finally to apoptotic cell death in elderly animal [35]. Glutathione-SH (GSH) antioxidants system is also indispensable for the cellular detoxification of ROS in cells. It has been shown a direct association between reduced levels of the GSH by severe oxidative stress and quick up-regulation of hemeoxygenase-1 (HO-1) in a different kind of cells [36, 37]. Eventually, increased HO-1 via the decrease of antioxidant defense systems appears to be as important as ROS production in the aging cells. Interestingly, BTG1 may also cause senescence by reducing mitochondrial membrane potential [38]. In addition, BTG1 overexpression may also induce G2/M arrest, differentiation, and senescence in a cell line cells [39]. Similarly, BTG2, another member of APRO family, is involved in a variety of biological processes including cell differentiation and cellular senescence, and its expression is deeply regulated by p53 [40]. However, oxidative stress upregulates the BTG2 expression via ROS-NFKB signaling cascade independent of p53 implying that it could be involved in mediating several biological phenotypes depending on the cellular context [40]. BTG2 may regulate posttranslational modification of p53 as contrasting to inhibiting sirtuin 1 (SIRT1) and B-cell/CLL lymphoma 2 (Bcl2) expression [41]. In addition, the expression of TOB1 also increases with cellular senescence [42].

\section{ROS, cancer and APRO family}

Hypoxia may play an imperative role in cancer cells-microenvironment [43]. During deprivation of enough oxygen supply, cells cannot keep adequate antioxidant capability resulting in increased ROS levels [44]. Hence, cells must adjust to the consequences of reduced oxygen availability. ROS levels may activate redox-sensitive transcription factors that can enhance tumor formation [45]. It is possible that increased ROS levels, brought 
during chronic inflammation, may stimulate aberrant self-renewal in tumor cells [46, 47] (Figure 1). Tumor cells frequently overexpress catalase and produce enormous concentrations of hydrogen peroxide [48]. The tumor cell itself escapes the toxic action of hydrogen peroxide then destroys neighboring healthy normal cells [49]. Increased ROS is responsible for the oxidation of negative feedback loop and hence control the actions of other signaling pathways in tumor cells growth by the PI3K/AKT pathway [50]. Cell survival is also promoted by the oxidation and inactivation of the negative regulators of PI3K/AKT signaling [51]. Generation of ROS in cancer cells may also lead to the inactivation of phosphatase and tensin homologue deleted on chromosome 10 (PTEN) that leads to an increase in PI3K/AKT signaling which promotes tumor cell-proliferation [52]. PTEN has been found to be inactivated by hydrogen peroxide in a variety of cancers [53]. The major ROS-regulated hypoxia inducible factor (HIF) may downregulate mammalian target of rapamycin (mTOR) which is a key regulator of cell growth by controlling cell metabolism, mRNA translation, and control of autophagy [54]. The tumor suppressor genes in cells may produce proteins that play important roles as antioxidants [55]. For instance, p53 could regulate the expression of various antioxidant enzymes including catalase and SOD2 thereby decreasing ROS accumulation [56]. It seems that induction of these p53 target genes is a conserved expression response to oxidative stress in different cells or organs. As p53 is deleted and/or mutated in almost all cancers, relationship between ROS accumulation and oncogenic signaling has been shown [57]. Amazingly, BTG2 is often downregulated in several types of cancer [58]. High-level BTG2 protein expression may correlate with prolonged survival in patients with breast cancer [59]. In addition, cytoplasmic TOB1 expression has been important in angiogenesis and cell differentiation within gastric cancer and may be used as a potential prognostic marker of the cancer [60]. Likewise, significant prognostic effects of the several APRO family have been found in lung adenocarcinoma [61]. Potential correlations between some APRO family members and survival outcomes are also observed in ovarian, colorectal and brain cancer [61]. Genomic profiling of B-cell leukemia and lymphoma has pointed BTG1 towards a role of tumor suppressors, since BTG1 is frequently deleted and/or mutated in these malignancies [62, 63]. Additionally, exosome-derived BTG1 protein is a potential biomarker for the prognosis in patients with lung cancer [64]. These findings have suggested that APRO genes and/or proteins might work as potential tools for cancer therapy. In fact, tumor growth suppression by adenovirus-mediated introduction of TOB1 in pancreatic cancer had been described suggesting a medical application for chemotherapy-resistant cancer [65].

\section{APRO family and ROS}

The APRO family anti-proliferative genes are characterized in immediate early growth responsive genes [66]. The gene products include pheochromocytoma cell 3 (PC3)/TIS21/BTG2, BTG1, TOB1, TOB2, abundant in neuroepithelium area (ANA)/BTG3, PC3B and others [66]. These APRO family proteins have been described to participate in diverse human diseases, which have also been implicated in a variety of cellular processes including cell-division, DNA repair, and mRNA stability [67]. Accordingly, APRO family members therefore may be involved in physiological and/or pathological processes including cell proliferation, cell differentiation, apoptosis/programmed cell death, and acting as potent tumor suppressors [67]. As for the regulation of ROS accumulation, BTG2 has been shown to render cancer cells more sensitive to doxorubicin treatment by upregulating SOD2 expression without regulating any other ROS scavengers [68]. BTG2 mediates crosstalk between PI3K/AKT and NF- $\mathrm{KB}$ pathways, which regulates p53-independent induction of G2/M arrest both within normal and cancer cells [68]. In addition, BTG2 enhances the G2/M arrest along with reduction of hydrogen peroxide levels [68]. TOB1 antagonizes the PI3K/AKT signaling then induces cancer cell apoptosis by activating BAX protein and inhibiting the BCL2 expression [69]. The AKT/PTEN and the tumor suppressor p53 pathways have been proven to play a central role in regulating cell apoptosis by regulating the oxidative stress and/or ROS reducing [70]. In addition, TOB1 may reduce the phosphorylation of AKT, resulting in decreased protein expression of $\beta$-catenin, which in turn declines the expression of cyclin D1 and/or cyclin-dependent kinase-4 (CDK4) [71]. BTG1 overexpression along with cancer therapy-irradiation may be involved in inhibition of the PI3K/AKT signaling pathway, suggesting that BTG1 promoted ionizing radio-sensitivity of breast cancer cells [72]. Low-levels of limited local ROS play an important role as redox-signaling molecules in pathways such as PI3K/AKT/mTOR signaling involved in the maintenance 
of cellular homeostasis [73]. Consistently, induction of p53 overexpression may bring cellular senescence, autophagy and apoptosis, which are dependent on the regulation of the PI3K/AKT/mTOR pathway with APRO family proteins and/or excess ROS production [74].

\section{Speculation for the APRO-function-mechanisms}

Given the diverse roles attributed to APRO family proteins, the molecular biological mechanisms of APRO family proteins might be quite intricate. The N-terminal conserved APRO domain is a protein-protein interaction module, which is capable of binding to DNA-binding transcription factors as well as the CNOT7 and CNOT8 deadenylase subunits of the carbon catabolite repression 4-negative on TATA-less (CCR4-NOT) complex [75]. Several members of the APRO family are shown to be implicated in transcription in the nucleus and cytoplasmic mRNA deadenylation and its turnover [76] (Figure 2). Likewise, TOB1 can concomitantly interact with the poly(A) nuclease complex CCR4- chromatin assembly factor-1(CAF1) and the cytoplasmic poly(A)-binding protein, suggesting a role of TOB1 in linking the deadenylation [77]. In addition, TOB1 and TOB2 proteins contain an extra-long C-terminal domain with two poly(A)-binding protein (PABP)-interacting motif 2 (PAM2) motifs [78]. These TOB proteins can interact with CAF1 and PABP simultaneously, which promotes general deadenylation of mRNA [77]. The interaction of TOB with CAF1 and PABP promotes deadenylation by recruiting the CAF1-CCR4 deadenylase complex to the 3 ' end of mRNAs with a poly(A) tail [77]. Interestingly, the antiproliferative effects of TOB1 have been suggested to involve the CAF1-CCR4 deadenylase complex [79], suggesting that TOB proteins can exert their antiproliferative function by modulating the mRNA turnover [80]. BTG2 also interacts with CAF1 deadenylase through its APRO domain, a defining feature of APRO family, to control cell proliferation [81]. In fact, it has been shown that mRNA destabilization by BTG1 and BTG2 sustains cell quiescence [82].

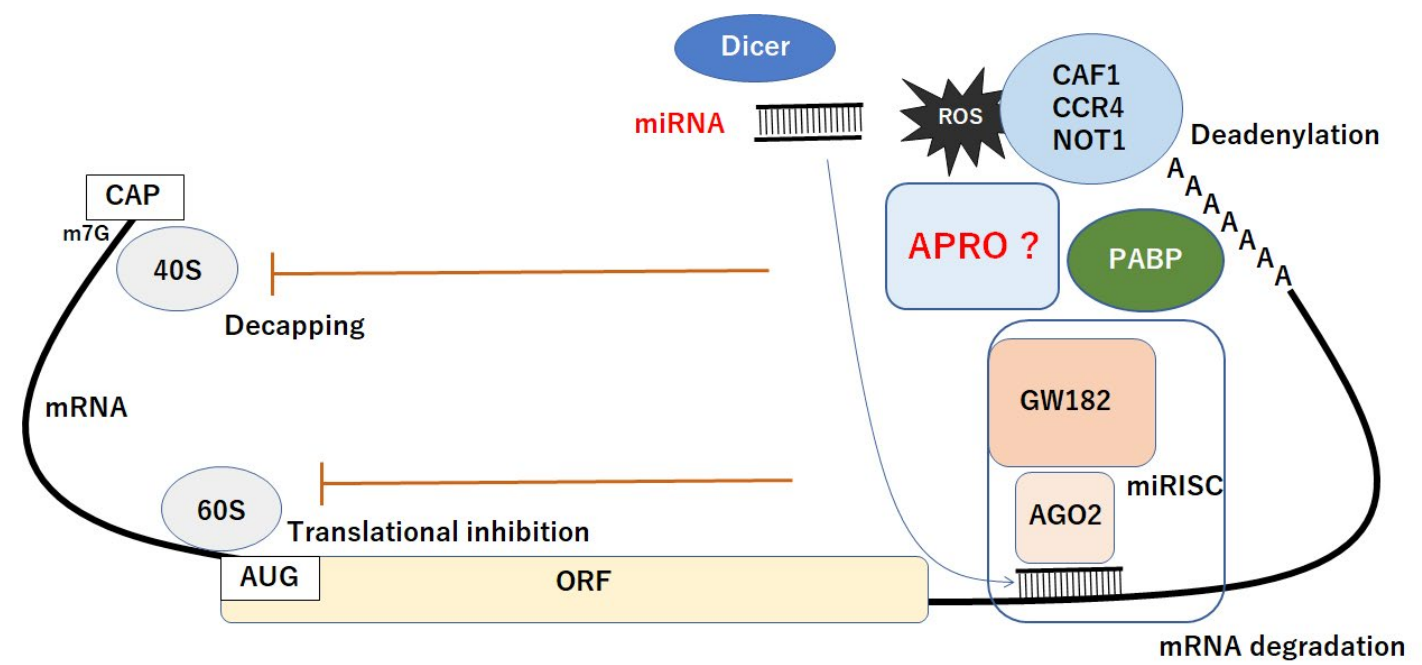

Figure 2. Schematic representation of miRNA-mediated functional inhibition of mRNA. The AGO2 protein interacts with GW182 constructing the miRISC complex, which may facilitate the deadenylation and mRNA degradation process by CAF1/CCR4/NOT1 with PABP and APRO complex. Consequently, the miRNA could play active roles in regulating posttranscriptional gene expression via the decapping, translational inhibition, deadenylation, and degradation of mRNA. The CAF1/CCR4/NOT1 complex is recruited to the 3'UTR of specific mRNAs through an interaction with PABP protein. APRO may also interact with PABP and recruits the CAF1/CCR4/NOT1 complex to initiate. Hammerhead represents inhibition. Note that some critical pathways have been omitted for clarity. CAF1: chromatin assembly factor-1; CAP: 5 ' capping structure of mRNA; ORF: open reading frame; miRISC: microRNA-induced silencing complex; AUG: initiation codon (ATG); PABP: poly A binding protein; GW182: an 182 kDa protein with multiple glycine/tryptophan (GW) repeats; AGO2: Argonaute2; ?: the phenomena have not yet been confirmed at present

MicroRNAs (miRNAs) inhibit mRNA expression by base pairing to the 3'UTR of target mRNAs, which consequently inhibits translation by initiating poly(A) tail deadenylation and mRNA destabilization [83]. The miRNA-mediated mRNA deadenylation occurs subsequent to initial translational inhibition, indicating a two-step mechanism of miRNA action, which serves to consolidate repression. The miRISC interacts with the PABP and the CAF1 and CCR4 deadenylases [84]. In addition, the miRNA-mediated deadenylation is dependent upon CAF1 activity and PABP, which serves as a miRNA coactivator [84]. Importantly, a core component of the miRISC could interact with PABP via its C-terminal region, which is required for 
miRNA-mediated deadenylation [84]. Accordingly, APRO family proteins could be a key modulator of miRNAs-function, since APRO family proteins have a potential to interact with CCR4/CAF1 complex (Figure 2). The CCR4/CAF1 complex has been shown a multifunctional regulator that plays important roles in multiple cellular processes in eukaryotes [85]. In addition, the expression of APRO family (TOB1, BTG1) may also be regulated by certain miRNAs $[24,86]$.

\section{Future perspectives}

There might be an unsolved paradigm in this field. Oxidative stress due to generation of ROS could cause damages to cellular proteins, lipids and DNAs, which is one of critical reasons responsible for carcinogenesis. Cancer or malignant tumor might have at least a cancer stem cell in its population, which can eventually render the disease immortality without senescence. Dicer, a key component of the miRNAs biogenesis, is a ribonuclease/helicase enzyme which involves in maturation of miRNAs. Dicer was found to be regulated by ROS/ NF-E2-related factor-2 (NRF2) interaction to contribute to activation of damaged-DNA-repair machinery. NRF2 may indirectly control the damaged-DNA-repair and miRNAs processing machinery through the crosstalk between NRF2 and Dicer [87]. In addition, lack of Dicer has been shown to enhance ROS production and oxidative stresses [88]. Furthermore, it has been shown that the lack of dicer activity in plant cells make them unable to control excessive ROS production [89].

The relationship between APRO molecules and carcinogenesis in a cell may be reminiscent of the relationship between cytotoxic T cells and cancer cells in a body called "immune check points" (Figure 3). We would like to suggest here tentatively to use a term "stemness check points" for the former relationship. However, this has to be confirmed precisely and would be a part of future rigorous research. The field of redox and stem cells medicine has blossomed in recent decades, but further evolvement will depend on linking more firmly distinct ROS and oxidative stress to pathophysiological processes. Given the crucial roles of ROS for pathophysiological functions, upcoming therapeutics should include vigilant modulation of the redox state. New approaches to modify the redox processes should be dynamic for improving health and quality of life (QOL) of individuals. Next generation medicine will likely include strategies for altering the redox environment with APRO being one of the key targets.

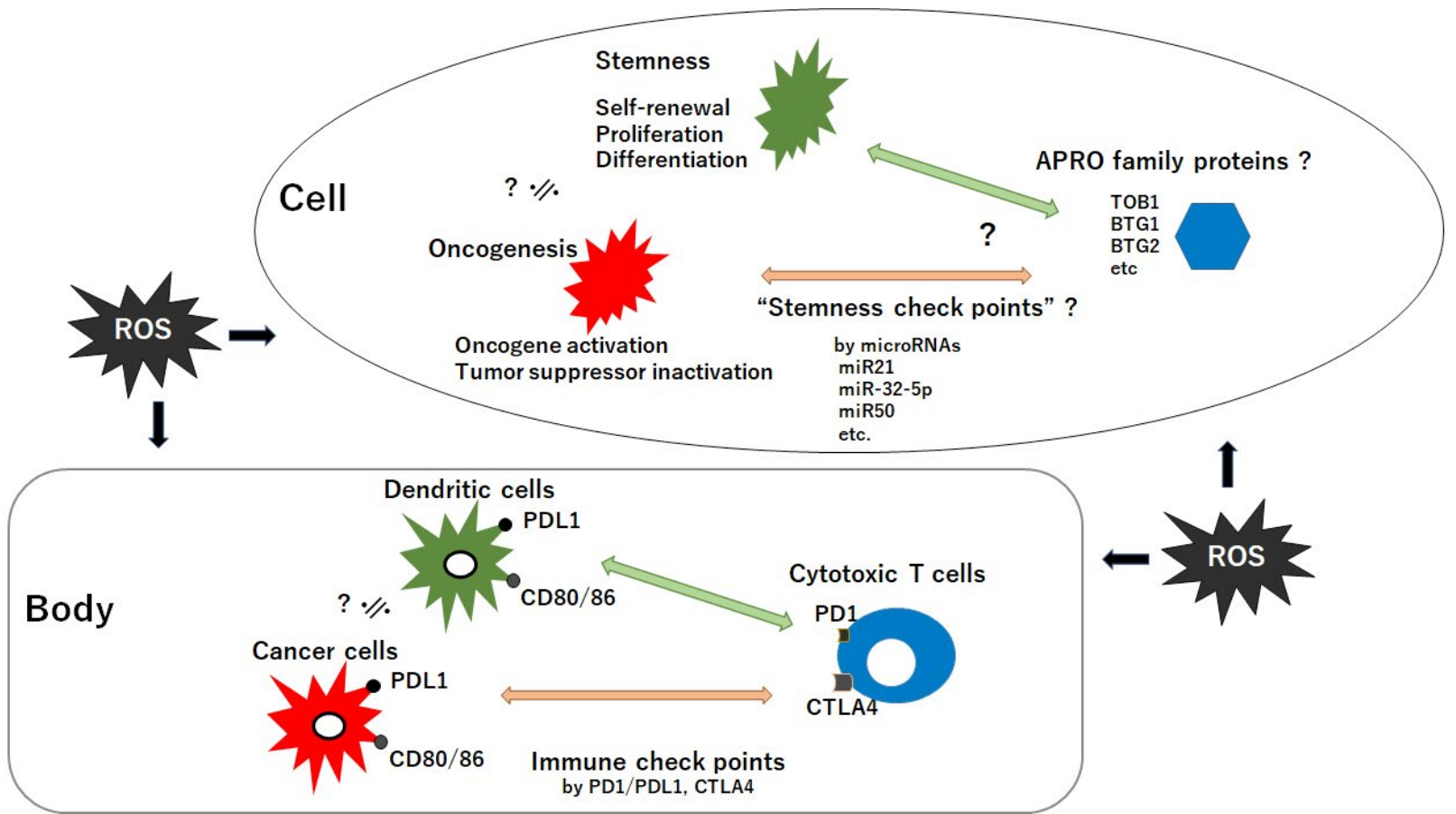

Figure 3. The relationship between tumor suppressor APRO family proteins and oncogenesis/stemness-homeostasis in a cell seems to look like the relationship between cytotoxic T-cells and cancer-cells/dendric-cells in a body. It could be hypothesized that the former may be regulated by "stemness checkpoints" microRNAs for targeting APRO family proteins, whereas the latter is regulated with "immune checkpoints" molecules such as PD1/PDL1 and CTLA4. ROS could affect both. CD: cluster of differentiation; PD: programmed cell death; PDL1: programmed cell death ligand 1; CTLA4: cytotoxic T-lymphocyte-associated protein $4 ; \fallingdotseq:$ approximately equal 


\section{Abbreviations}

APRO: anti-proliferative

APROs: anti-proliferative proteins

BTG: B-cell translocation gene

CAF1: chromatin assembly factor-1

CCR4: carbon catabolite repression 4

miRISC: microRNA-induced silencing complex

miRNAs: microRNAs

mRNA: messenger RNA

mTOR: mammalian target of rapamycin

NOT: negative on TATA-less

NRF2: NF-E2-related factor-2

PABP: poly(A)-binding protein

PI3K: phosphoinositide-3 kinase

PTEN: phosphatase and tensin homologue deleted on chromosome 10

ROS: reactive oxygen species

SOD: superoxide dismutase

TOB: transducer of ErbB-2

\section{Declarations}

Author contributions

YI and SM contributed conception of the study. YI, KT, NN, AT, YK and SM wrote sections of the manuscript. All authors contributed to manuscript revision, read, and approved the submitted version.

\section{Conflicts of interest}

The authors declare that they have no conflicts of interest.

Ethical approval

Not applicable.

Consent to participate

Not applicable.

\section{Consent of publication}

Not applicable.

Availability of data and materials

Not applicable.

\section{Funding}

Not applicable.

\section{Copyright}

(c) The Authors 2021.

\section{References}


1. Salehi B, Martorell M, Arbiser JL, Sureda A, Martins N, Maurya PK, et al. Antioxidants: positive or negative actors? Biomolecules. 2018;8:124.

2. Zerbinati C, Iuliano L. Cholesterol and related sterols autoxidation. Free Radic Biol Med. 2017;111:151-5.

3. Palka J, Oscilowska I, Szoka L. Collagen metabolism as a regulator of proline dehydrogenase/proline oxidase-dependent apoptosis/autophagy. Amino Acids. 2021; [Epub ahead of print].

4. De Gaetano A, Gibellini L, Zanini G, Nasi M, Cossarizza A, Pinti M. Mitophagy and oxidative stress: the role of aging. Antioxidants (Basel). 2021;10:794.

5. Wilson C, Muñoz-Palma E, González-Billault C. From birth to death: a role for reactive oxygen species in neuronal development. Semin Cell Dev Biol. 2018;80:43-9.

6. Shao L, Li H, Pazhanisamy SK, Meng A, Wang Y, Zhou D. Reactive oxygen species and hematopoietic stem cell senescence. Int J Hematol. 2011;94:24-32.

7. Farrow MA, Chumbler NM, Lapierre LA, Franklin JL, Rutherford SA, Goldenring JR, et al. Clostridium difficile toxin B-induced necrosis is mediated by the host epithelial cell NADPH oxidase complex. Proc Natl Acad Sci U S A. 2013;110:18674-9.

8. Chen S, Vurusaner B, Pena S, Thu CT, Mahal LK, Fisher EA, et al. Two-photon, ratiometric, quantitative fluorescent probe reveals fluctuation of peroxynitrite regulated by arginase 1. Anal Chem. 2021;93:10090-8.

9. Meijles DN, Zoumpoulidou G, Markou T, Rostron KA, Patel R, Lay K, et al. The cardiomyocyte "redox rheostat": redox signalling via the AMPK-mTOR axis and regulation of gene and protein expression balancing survival and death. J Mol Cell Cardiol. 2019;129:118-29.

10. Lim IK. TIS21 (/BTG2/PC3) as a link between ageing and cancer: cell cycle regulator and endogenous cell death molecule. J Cancer Res Clin Oncol. 2006;132:417-26.

11. Micheli L, Ceccarelli M, Farioli-Vecchioli S, Tirone F. Control of the normal and pathological development of neural stem and progenitor cells by the PC3/Tis21/Btg2 and Btg1 genes. J Cell Physiol. 2015;230:2881-90.

12. Paik WK, Kim S, Lim IK. Protein methylation and interaction with the antiproliferative gene, BTG2/TIS21/Pc3. Yonsei Med J. 2014;55:292-303.

13. Ahamad N, Singh BB. Calcium channels and their role in regenerative medicine. World J Stem Cells. 2021;13:260-80.

14. Ceccarelli M, D'Andrea G, Micheli L, Tirone F. Interaction between neurogenic stimuli and the gene network controlling the activation of stem cells of the adult neurogenic niches, in physiological and pathological conditions. Front Cell Dev Biol. 2020;8:211.

15. Zhang J, Lan T, Han X, Xu Y, Liao L, Xie L, et al. Improvement of ECM-based bioroot regeneration via $N$-acetylcysteine-induced antioxidative effects. Stem Cell Res Ther. 2021;12:202.

16. Yang J, Liang J, Zhu Y, Hu M, Deng L, Cui W, et al. Fullerol-hydrogel microfluidic spheres for in situ redox regulation of stem cell fate and refractory bone healing. Bioact Mater. 2021;6:4801-15.

17. Haval GA, Pekhale KD, Perween NA, Ghaskadbi SM, Ghaskadbi SS. Excess hydrogen peroxide inhibits head and foot regeneration in hydra by affecting DNA repair and expression of essential genes. J Biochem Mol Toxicol. 2020;34:e22577.

18. Rok J, Rzepka Z, Maszczyk M, Beberok A, Wrześniok D. Minocycline impact on redox homeostasis of normal human melanocytes HEMn-LP exposed to UVA radiation and hydrogen peroxide. Int J Mol Sci. 2021;22:1642.

19. Drehmer DL, de Aguiar AM, Brandt AP, Petiz L, Cadena SM, Rebelatto CK, et al. Metabolic switches during the first steps of adipogenic stem cells differentiation. Stem Cell Res. 2016;17:413-21. 
20. Ecker $V$, Stumpf $M$, Brandmeier $L$, Neumayer $T$, Pfeuffer $L$, Engleitner $T$, et al. Targeted PI3K/AKT-hyperactivation induces cell death in chronic lymphocytic leukemia. Nat Commun. 2021;12:3526.

21. Pasha A, Calvani M, Favre C. beta3-adrenoreceptors as ROS balancer in hematopoietic stem cell transplantation. Int J Mol Sci. 2021;22:2835.

22. Olivier C, Oliver L, Lalier L, Vallette FM. Drug resistance in glioblastoma: the two faces of oxidative stress. Front Mol Biosci. 2021;7:620677.

23. Chen Y, Wang C, Wu J, Li L. BTG/Tob family members Tob1 and Tob2 inhibit proliferation of mouse embryonic stem cells via Id3 mRNA degradation. Biochem Biophys Res Commun. 2015;462:208-14.

24. Gao Y, Zhang Y, Lu Y, Wang Y, Kou X, Lou Y, et al. TOB1 deficiency enhances the effect of bone marrow-derived mesenchymal stem cells on tendon-bone healing in a rat rotator cuff repair model. Cell Physiol Biochem. 2016;38:319-29.

25. Farioli-Vecchioli S, Micheli L, Saraulli D, Ceccarelli M, Cannas S, Scardigli R, et al. Btg1 is required to maintain the pool of stem and progenitor cells of the dentate gyrus and subventricular zone. Front Neurosci. 2012;6:124.

26. Choi SC, Choi JH, Park CY, Ahn CM, Hong SJ, Lim DS. Nanog regulates molecules involved in stemness and cell cycle-signaling pathway for maintenance of pluripotency of P19 embryonal carcinoma stem cells. J Cell Physiol. 2012;227:3678-92.

27. Kim BC, Ryu MS, Oh SP, Lim IK. TIS21/(BTG2) negatively regulates estradiol-stimulated expansion of hematopoietic stem cells by derepressing Akt phosphorylation and inhibiting mTOR signal transduction. Stem Cells. 2008;26:2339-48.

28. Mastrorilli V, Scopa C, Saraulli D, Costanzi M, Scardigli R, Rouault JP, et al. Physical exercise rescues defective neural stem cells and neurogenesis in the adult subventricular zone of Btg1 knockout mice. Brain Struct Funct. 2017;222:2855-76.

29. Periyasamy P, Shinohara T. Age-related cataracts: role of unfolded protein response, $\mathrm{Ca}^{2+}$ mobilization, epigenetic DNA modifications, and loss of Nrf2/Keap1 dependent cytoprotection. Prog Retin Eye Res. 2017;60:1-19.

30. Zhu L, Dai Y, Gao L, Zhao Q. Tumor microenvironment-modulated nanozymes for NIR-II-triggered hyperthermia-enhanced photo-nanocatalytic therapy via disrupting ROS homeostasis. Int J Nanomedicine. 2021;16:4559-77.

31. LiX, Rommelaere S, Kondo S, Lemaitre B. Renal purge of hemolymphatic lipids prevents the accumulation of ROS-induced inflammatory oxidized lipids and protects drosophila from tissue damage. Immunity. 2020;52:374-87.e6.

32. Suzuki YJ, Hao JJ. Results supporting the concept of the oxidant-mediated protein amino acid conversion, a naturally occurring protein engineering process, in human cells. F1000Res. 2017;6:594.

33. Pleshakova OV, Kutsyi MP, Sukharev SA, Sadovnikov VB, Gaziev AI. Study of protein carbonyls in subcellular fractions isolated from liver and spleen of old and gamma-irradiated rats. Mech Ageing Dev. 1998;103:45-55.

34. Rudzińska M, Parodi A, Balakireva AV, Chepikova OE, Venanzi FM, Zamyatnin AA Jr. Cellular aging characteristics and their association with age-related disorders. Antioxidants (Basel). 2020;9:94.

35. Lin TH, Chiu YJ, Lin CH, Lin CY, Chao CY, Chen YC, et al. Exploration of multi-target effects of 3-benzoyl-5-hydroxychromen-2-one in Alzheimer's disease cell and mouse models. Aging Cell. 2020;19:e13169.

36. Wu CT, Deng JS, Huang WC, Shieh PC, Chung MI, Huang GJ. Salvianolic acid C against acetaminophen-induced acute liver injury by attenuating inflammation, oxidative stress, and apoptosis through inhibition of the Keap1/Nrf2/HO-1 signaling. Oxid Med Cell Longev. 2019;2019:9056845. 
37. Rasquel-Oliveira FS, Manchope MF, Staurengo-Ferrari L, Ferraz CR, Saraiva-Santos T, Zaninelli TH, et al. Hesperidin methyl chalcone interacts with NFKB Ser276 and inhibits zymosan-induced joint pain and inflammation, and RAW 264.7 macrophage activation. Inflammopharmacology. 2020;28:979-92.

38. Zhao S, Chen SR, Yang XF, Shen DF, Takano Y, Su RJ, et al. BTG1 might be employed as a biomarker for carcinogenesis and a target for gene therapy in colorectal cancers. Oncotarget. 2017;8:7502-20.

39. Zheng HC, Li J, Shen DF, Yang XF, Zhao S, Wu YZ, et al. BTG1 expression correlates with pathogenesis, aggressive behaviors and prognosis of gastric cancer: a potential target for gene therapy. Oncotarget. 2015;6:19685-705.

40. Imran M, Lim IK. Regulation of Btg2 (/TIS21/PC3) expression via reactive oxygen species-protein kinase C-NFKB pathway under stress conditions. Cell Signal. 2013;25:2400-12.

41. Choi OR, Ryu MS, Lim IK. Shifting p53-induced senescence to cell death by TIS21(/BTG2/Pc3) gene through posttranslational modification of p53 protein. Cell Signal. 2016;28:1172-85.

42. Yuan J, Cao JY, Tang ZL, Wang N, Li K. Molecular characterization of Tob1 in muscle development in pigs. Int J Mol Sci. 2011;12:4315-26.

43. Ikeda Y, Nagase N, Tsuji A, Taniguchi K, Kitagishi Y, Matsuda S. Comprehension of the relationship between autophagy and reactive oxygen species for superior cancer therapy with histone deacetylase inhibitors. Oxygen 2021:1:22-31.

44. Yadav AK, Yadav PK, Chaudhary GR, Tiwari M, Gupta A, Sharma A, et al. Autophagy in hypoxic ovary. Cell Mol Life Sci. 2019;76:3311-22.

45. Kumari Kanchan R, Tripathi C, Singh Baghel K, Kumar Dwivedi S, Kumar B, Sanyal S, et al. Estrogen receptor potentiates mTORC2 signaling in breast cancer cells by upregulating superoxide anions. Free Radic Biol Med. 2012;53:1929-41.

46. Wu S, Lu H, Bai Y. Nrf2 in cancers: a double-edged sword. Cancer Med. 2019;8:2252-67.

47. Rinkenbaugh AL, Baldwin AS. The NF-kappaB pathway and cancer stem cells. Cells. 2016;5:16.

48. Bai J, Rodriguez AM, Melendez JA, Cederbaum AI. Overexpression of catalase in cytosolic or mitochondrial compartment protects HepG2 cells against oxidative injury. J Biol Chem. 1999;274:26217-24.

49. Ming J, Zhu T, Yang W, Shi Y, Huang D, Li J, et al. Pd@Pt-GOx/HA as a novel enzymatic cascade nanoreactor for high-efficiency starving-enhanced chemodynamic cancer therapy. ACS Appl Mater Interfaces. 2020;12:51249-62.

50. Wang P, Zhao L, Gong S, Xiong S, Wang J, Zou D, et al. HIF1alpha/HIF2alpha-Sox2/Klf4 promotes the malignant progression of glioblastoma via the EGFR-PI3K/AKT signalling pathway with positive feedback under hypoxia. Cell Death Dis. 2021;12:312.

51. Srinivas KP, Viji R, Dan VM, Sajitha IS, Prakash R, Rahul PV, et al. DEPTOR promotes survival of cervical squamous cell carcinoma cells and its silencing induces apoptosis through downregulating PI3K/AKT and by up-regulating p38 MAP kinase. Oncotarget. 2016;7:24154-71.

52. Cai B, Liu M, Li J, Xu D, Li J. Cigarette smoke extract amplifies NADPH oxidase-dependent ROS production to inactivate PTEN by oxidation in BEAS-2B cells. Food Chem Toxicol. 2021;150:112050.

53. Chetram MA, Don-Salu-Hewage AS, Hinton CV. ROS enhances CXCR4-mediated functions through inactivation of PTEN in prostate cancer cells. Biochem Biophys Res Commun. 2011;410:195-200.

54. Cosin-Roger J, Simmen S, Melhem H, Atrott K, Frey-Wagner I, Hausmann M, et al. Hypoxia ameliorates intestinal inflammation through NLRP3/mTOR downregulation and autophagy activation. Nat Commun. 2017;8:98.

55. Liu X, Fan L, Lu C, Yin S, Hu H. Functional role of p53 in the regulation of chemical-induced oxidative stress. Oxid Med Cell Longev. 2020;2020:6039769.

56. Bravard A, Petridis F, Luccioni C. Modulation of antioxidant enzymes p21WAF1 and p53 expression during proliferation and differentiation of human melanoma cell lines. Free Radic Biol Med. 1999;26:1027-33. 
57. Tessoulin B, Descamps G, Dousset C, Amiot M, Pellat-Deceunynck C. Targeting oxidative stress with auranofin or Prima- $1^{\text {Met }}$ to circumvent p53 or Bax/Bak deficiency in myeloma cells. Front Oncol. 2019;9:128.

58. Shen S, Zhang R, Guo Y, Loehrer E, Wei Y, Zhu Y, et al. A multi-omic study reveals BTG2 as a reliable prognostic marker for early-stage non-small cell lung cancer. Mol Oncol. 2018;12:913-24.

59. Möllerström E, Kovács A, Lövgren K, Nemes S, Delle U, Danielsson A, et al. Up-regulation of cell cycle arrest protein BTG2 correlates with increased overall survival in breast cancer, as detected by immunohistochemistry using tissue microarray. BMC Cancer. 2010;10:296.

60. Zhang SQ, Sun KK, Wu XY, Zhong N, Zhao H, Li DC. Clinicopathological significance of cytoplasmic transducer of ErbB2. 1 expression in gastric cancer. Mol Med Rep. 2015;12:1177-82.

61. Bai Y, Qiao L, Xie N, Shi Y, Liu N, Wang J. Expression and prognosis analyses of the Tob/BTG antiproliferative (APRO) protein family in human cancers. PLoS One. 2017;12:e0184902.

62. Moafi A, Zojaji A, Salehi R, Najafi Dorcheh S, Rahgozar S. The correlation between Pax5 deletion and patients survival in Iranian children with precursor B-cell acute lymphocytic leukemia. Cell Mol Biol (Noisy-le-grand). 2017;63:19-22.

63. Rimokh R, Rouault JP, Wahbi K, Gadoux M, Lafage M, Archimbaud E, et al. A chromosome 12 coding region is juxtaposed to the MYC protooncogene locus in a $\mathrm{t}(8 ; 12)(\mathrm{q} 24 ; \mathrm{q} 22)$ translocation in a case of B-cell chronic lymphocytic leukemia. Genes Chromosomes Cancer. 1991;3:24-36.

64. Wan L, Chen X, Deng J, Zhang S, Tu F, Pei H, et al. Plasma exosome-derived B-cell translation gene 1: a predictive marker for the prognosis in patients with non-small cell lung cancer. J Cancer. 2021;12:1538-47.

65. Yanagie H, Tanabe T, Sumimoto H, Sugiyama H, Matsuda S, Nonaka Y, et al. Tumor growth suppression by adenovirus-mediated introduction of a cell-growth-suppressing gene tob in a pancreatic cancer model. Biomed Pharmacother. 2009;63:275-86.

66. Matsuda S, Rouault J, Magaud J, Berthet C. In search of a function for the TIS21/PC3/BTG1/TOB family. FEBS Lett. 2001;497:67-72.

67. Yuniati L, Scheijen B, van der Meer LT, van Leeuwen FN. Tumor suppressors BTG1 and BTG2: beyond growth control. J Cell Physiol. 2019;234:5379-89.

68. Sundaramoorthy S, Ryu MS, Lim IK. B-cell translocation gene 2 mediates crosstalk between PI3K/Akt1 and NFkappaB pathways which enhances transcription of MnSOD by accelerating IkappaBalpha degradation in normal and cancer cells. Cell Commun Signal. 2013;11:69.

69. Lee HS, Kundu J, Kim RN, Shin YK. Transducer of ERBB2.1 (TOB1) as a tumor suppressor: a mechanistic perspective. Int J Mol Sci. 2015;16:29815-28.

70. Matsuda S, Nakagawa Y, Kitagishi Y, Nakanishi A, Murai T. Reactive oxygen species, superoxide dimutases, and PTEN-p53-AKT-MDM2 signaling loop network in mesenchymal stem/stromal cells regulation. Cells. 2018;7:36.

71. Kundu J, Wahab SM, Kundu JK, Choi YL, Erkin OC, Lee HS, et al. Tob1 induces apoptosis and inhibits proliferation, migration and invasion of gastric cancer cells by activating Smad4 and inhibiting beta-catenin signaling. Int J Oncol. 2012;41:839-48.

72. Zhu R, Li W, Xu Y, Wan J, Zhang Z. Upregulation of BTG1 enhances the radiation sensitivity of human breast cancer in vitro and in vivo. Oncol Rep. 2015;34:3017-24.

73. Checa J, Aran JM. Reactive oxygen species: drivers of physiological and pathological processes. J Inflamm Res. 2020;13:1057-73.

74. Simabuco FM, Morale MG, Pavan ICB, Morelli AP, Silva FR, Tamura RE. p53 and metabolism: from mechanism to therapeutics. Oncotarget. 2018;9:23780-823. 
75. Doidge R, Mittal S, Aslam A, Winkler GS. The anti-proliferative activity of BTG/TOB proteins is mediated via the Caf1a (CNOT7) and Caf1b (CNOT8) deadenylase subunits of the Ccr4-not complex. PLoS One. 2012;7:e51331.

76. Winkler GS. The mammalian anti-proliferative BTG/Tob protein family. J Cell Physiol. 2010;222:66-72.

77. Ezzeddine N, Chang TC, Zhu W, Yamashita A, Chen CY, Zhong Z, et al. Human TOB, an antiproliferative transcription factor, is a poly(A)-binding protein-dependent positive regulator of cytoplasmic mRNA deadenylation. Mol Cell Biol. 2007;27:7791-801.

78. Lim NS, Kozlov G, Chang TC, Groover O, Siddiqui N, Volpon L, et al. Comparative peptide binding studies of the PABC domains from the ubiquitin-protein isopeptide ligase HYD and poly(A)-binding protein. Implications for HYD function. J Biol Chem. 2006;281:14376-82.

79. Horiuchi M, Takeuchi K, Noda N, Muroya N, Suzuki T, Nakamura T, et al. Structural basis for the antiproliferative activity of the Tob-hCaf1 complex. J Biol Chem. 2009;284:13244-55.

80. Ezzeddine N, Chen CY, Shyu AB. Evidence providing new insights into TOB-promoted deadenylation and supporting a link between TOB's deadenylation-enhancing and antiproliferative activities. Mol Cell Biol. 2012;32:1089-98.

81. Stupfler B, Birck C, Séraphin B, Mauxion F. BTG2 bridges PABPC1 RNA-binding domains and CAF1 deadenylase to control cell proliferation. Nat Commun. 2016;7:10811.

82. Hwang SS, Lim J, Yu Z, Kong P, Sefik E, Xu H, et al. mRNA destabilization by BTG1 and BTG2 maintains T cell quiescence. Science. 2020;367:1255-60.

83. Flamand MN, Wu E, Vashisht A, Jannot G, Keiper BD, Simard MJ, et al. Poly(A)-binding proteins are required for microRNA-mediated silencing and to promote target deadenylation in C. elegans. Nucleic Acids Res. 2016;44:5924-35.

84. Fabian MR, Mathonnet G, Sundermeier T, Mathys H, Zipprich JT, Svitkin YV, et al. Mammalian miRNA RISC recruits CAF1 and PABP to affect PABP-dependent deadenylation. Mol Cell. 2009;35:868-80.

85. Dai Y, Cao Z, Huang L, Liu S, Shen Z, Wang Y, et al. CCR4-Not complex subunit Not2 plays critical roles in vegetative growth, conidiation and virulence in watermelon Fusarium wilt pathogen Fusarium oxysporum f. sp. niveum. Front Microbiol. 2016;7:1449.

86. Cheng Q Li Q Xu L, Jiang H. Exosomal microRNA-301a-3p promotes the proliferation and invasion of nasopharyngeal carcinoma cells by targeting BTG1 mRNA. Mol Med Rep. 2021;23:328.

87. Nabih HK. Crosstalk between NRF2 and Dicer through metastasis regulating MicroRNAs; mir-34a, mir-200 family and mir-103/107 family. Arch Biochem Biophys. 2020;686:108326.

88. Singh Y, Zhou Y, Zhang S, Abdelazeem KNM, Elvira B, Salker MS, et al. Enhanced reactive oxygen species production, acidic cytosolic $\mathrm{pH}$ and upregulated $\mathrm{Na}^{+} / \mathrm{H}^{+}$exchanger (NHE) in Dicer deficient $\mathrm{CD} 4^{+} \mathrm{T}$ cells. Cell Physiol Biochem. 2017;42:1377-89.

89. Suzuki T, Ikeda S, Kasai A, Taneda A, Fujibayashi M, Sugawara K, et al. RNAi-mediated down-regulation of Dicer-like 2 and 4 changes the response of 'moneymaker' tomato to potato spindle tuber viroid infection from tolerance to lethal systemic necrosis, accompanied by up-regulation of miR398, 398a-3p and production of excessive amount of reactive oxygen species. Viruses. 2019;11:344. 